\title{
Dark matter in the Galactic center
}

\author{
Tim Linden \\ The Kavli Institute for Cosmological Physics, University of Chicago \\ Chicago, IL 60637 USA \\ email: trlinden@uchicago.edu
}

\begin{abstract}
In addition to boasting the highest density of baryonic matter in our galaxy, the center of the Milky Way is also believed to contain an extremely high density of dark matter particles. While dark matter is expected to be gravitationally subdominant to baryons near the Galactic center, many models allow for the annihilation of dark matter into standard model particles, a phenomenon which could be a significant source of high energy radiation in the Galactic center region. In fact, standard models of the dark matter density distribution and annihilation spectrum predict that dark matter at the Galactic center would (1) produce the brightest flux from dark matter annihilation of any region in the sky, and (2) contribute a significant portion of the total $\gamma$-ray luminosity observed within several degrees of the Galactic center by the Fermi/LAT. This makes the effort to understand and differentiate the morphologies and spectral features of dark matter and astrophysical $\gamma$-ray emission at the Galactic center potentially rewarding. Here, I will summarize the recent developments in indirect searches for dark matter annihilation at the Galactic center, and discuss several of the difficulties in producing accurate models of the high energy astrophysical emission. Finally, I will comment on current efforts to produce multi-wavelength models which better constrain or indicate a dark matter signal at the Galactic center.
\end{abstract}

\section{The density of dark matter in the Galactic center}

Recently large scale simulations have been employed to help understand the density profiles of dark matter halos in our universe. An early, seminal paper by Navarro, Frenk and White showed that the dark matter profile stemming from simulations could be fit by a simple, power-law model over several decades in radius (Navarro et al. (1996)). More recent simulations by Via Lactea II and Aquarius are best fit by slightly more complicated (but qualitatively similar) dark matter density profiles which span from the simulation resolution (often $\gtrsim 100 \mathrm{pc}$ ) up to the virial radius of the dark matter halo (Diemand et al. (2008), Navarro et al. (2004)). A nearly universal prediction of these simulations is the fact that the highest luminosity from models of annihilating dark matter will come from the center of the Milky Way galaxy, due to its combination of a high dark matter density and its relative proximity to Earth compared to extragalactic sources.

However, these simulations do not include baryonic matter, and thus may not be applicable to observed galaxies, which are dominated in the center by the baryonic density. With hydrodynamical simulations including baryons still in their infancy, there is currently a debate within the literature as to the net effect of baryons on the dark matter density profile in regions with significant baryon densities. Simulations by Gnedin et al. (2011) indicate that the efficiency of baryonic cooling in the centers of galaxies steepens the galactic potential, pulling dark matter particles in closer to the Galactic center - a process called adiabatic contraction. However, simulations by Governato et al. (2011) find that rapid, supernova driven gaseous outflows transfer energy to the dark matter component and produce halos which are less peaked in the Galactic center compared to dark matter only simulations. 


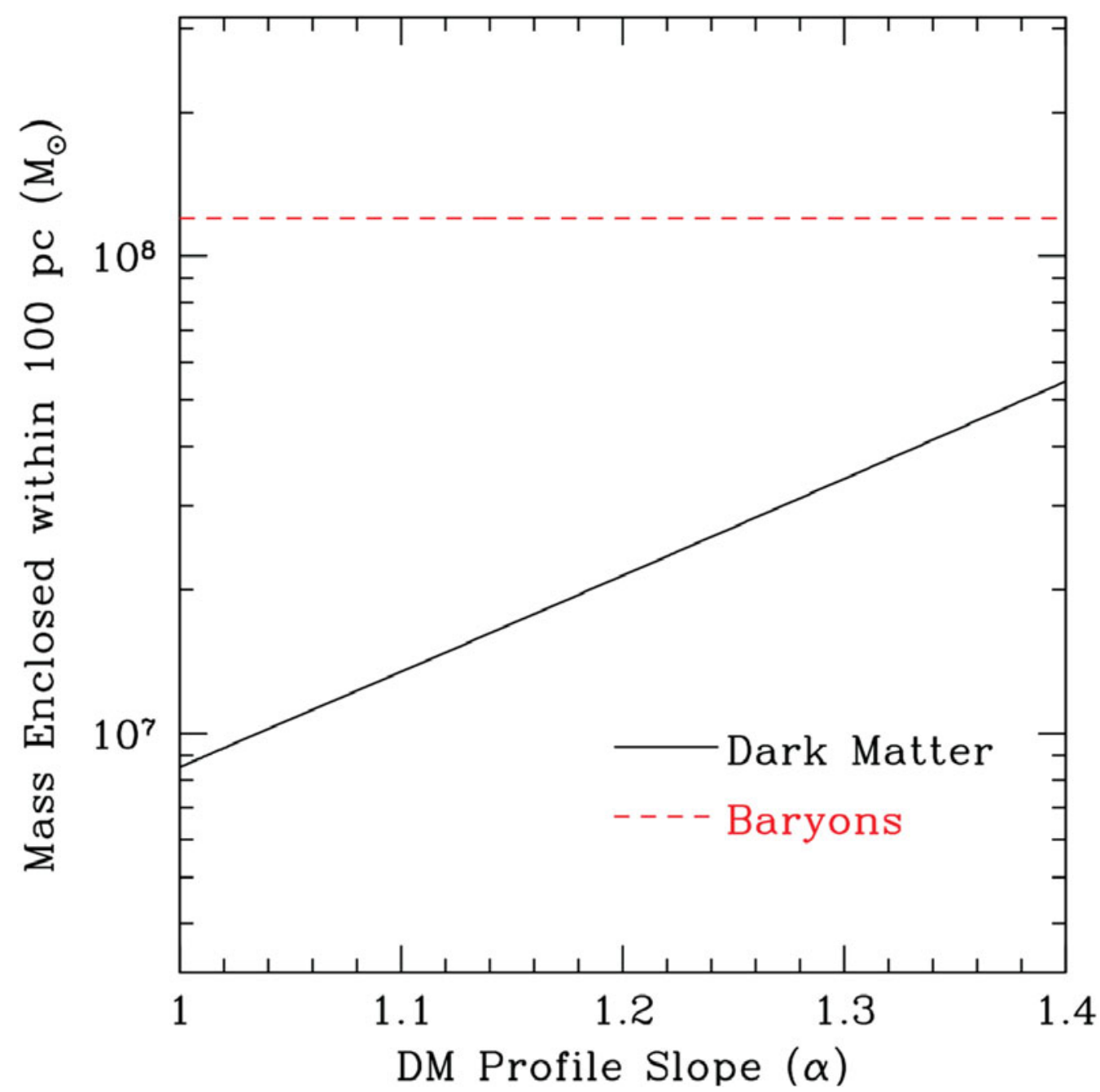

Figure 1. The dark matter and baryonic masses enclosed within 100 pc of the Galactic center for various choices of the slope of the dark matter profile (as given by Equation 1.1), compared to an enclosed baryonic mass based on the models of McMillan (2011). The local density of dark matter is assumed to be $0.3 \mathrm{GeV} \mathrm{cm}^{-3}$ and the scale radius for the dark matter density distribution is assumed to be $20 \mathrm{kpc}$. [A COLOR VERSION IS AVAILABLE ONLINE.]

In order to generalize our work with regards to these results, we will adopt a generalized version of the NFW profile following the functional form:

$$
\rho_{N F W}(r)=\rho_{0}\left(\frac{r}{r_{s}}\right)^{-\alpha}\left(1+\frac{r}{r_{s}}\right)^{-3+\alpha}
$$

where $\alpha$ slope of the dark matter density profile near the Galactic center, while the slope far from the center of the dark matter halo is $-3, \mathrm{r}_{s}$ is the scaling radius at which the turnover in the power-law slope occurs, and $\rho_{0}$ is the dark matter density at this critical point. This corresponds to the traditional profile given by Navarro et al. (1996) in the case that $\alpha=1$. In the case of the Milky Way halo, the value of $\rho_{0}$ is set by observations of the local dark matter density near the solar position, with observations pointing towards $\rho_{\odot} \sim 0.2-0.5 \mathrm{GeV} \mathrm{cm}^{-3}$ (Iocco et al. 2011). 
In nearly all cases, the gravitational potential from dark matter near the Galactic center negligible, compared to that from observed baryons. Given the density profile in Equation 1.1, the dark matter mass enclosed within $100 \mathrm{pc}$ of the Galactic center is approximately $8.5 \times 10^{6} \mathrm{M}_{\odot}$ for $\alpha=1.0$, compared to a baryonic density of $1.2 \times 10^{8} \mathrm{M}_{\odot}$ within this region, which is obtained by employing a Hernquist profile based on the model of McMillan (2011). In Figure 1 we show the enclosed mass within 100 pc of the Galactic center for various choices of the dark matter density profile $(\alpha)$, showing that even in fairly extreme cases, the dark matter contribution is subdominant to baryons.

\section{Dark matter particle physics}

While cosmological observations show that dark matter dominates the matter density of the universe, the nature of the particle (or particles) which make up the dark matter has not yet been determined. Known astrophysical objects, such as black holes, brown dwarfs, or smaller structures, have been ruled out by optical and infrared observations. Known weakly interacting particles, such as neutrinos, have been ruled out by simulations which show that relativistic dark matter particles would not clump on the correct scales to produce the observed dark matter power spectrum. The search for dark matter has thus moved on to searches for novel particles with the correct particle physics and gravitational properties. Early on, it was noted that a particle having both a mass on the weak scale $\sim 100 \mathrm{GeV}$, as well as a weak interaction cross-section in the early universe $\left(\sigma \mathrm{v} \sim 3.0 \times 10^{-26} \mathrm{~cm}^{3} \mathrm{~s}^{-1}\right)$ would naturally obtain the correct relic density of dark matter in the universe (Steigman (1979)). Another possible hint from particle physics came from the concept of supersymmetry. If supersymmetric particles were forbidden from decaying to pairs of standard model particles (by some conserved parity), then the lightest supersymmetric particle would be stable, and may be the dark matter Pagels \& Primack (1982). This model, where the lightest supersymmetric particle can interact through the weak force (and thus obtains a mass on the order 10-1000 GeV, remains the best theoretically motivated model for the dark matter particle. In what follows we will work within this context and consider a search for a weakly interacting massive particle (WIMP) dark matter at the center of the Milky Way galaxy.

\section{Constraints on dark matter from the Galactic center}

If dark matter particles are weakly interacting in the early universe, these interactions should continue (albeit at a highly suppressed rate) today, and may be visible in situations where two dark matter particles annihilate to produce a jet of highly energetic standard model particles. These products of these annihilations (including photons) could be observable in any region of space where the dark matter particle density is very high. Within this paradigm the dark matter annihilation luminosity in any region of space can be written as:

$$
\Phi_{D M}=\frac{1}{2}\left[\frac{<\sigma v>}{M_{D M}^{2}} \int_{0}^{m_{D M}} \frac{d N}{d E} d E\right]\left[\int \frac{\rho_{D M}^{2}(\vec{x})}{r^{2}} d V\right]
$$

where $\langle\sigma v\rangle$ is the WIMP annihilation rate $\frac{d N}{d E}$ is the dark matter annihilation spectrum for photons and $\mathrm{M}_{D M}$ is the dark matter particle mass. The $\frac{1}{2}$ resolves the double counting of dark matter particles in the annihilation event, and the local dark matter density at a position in space is given by $\rho_{D M}^{2}(\vec{x})$. It is worth noting that the first set of brackets includes only particle physics assumptions, while the second set includes 

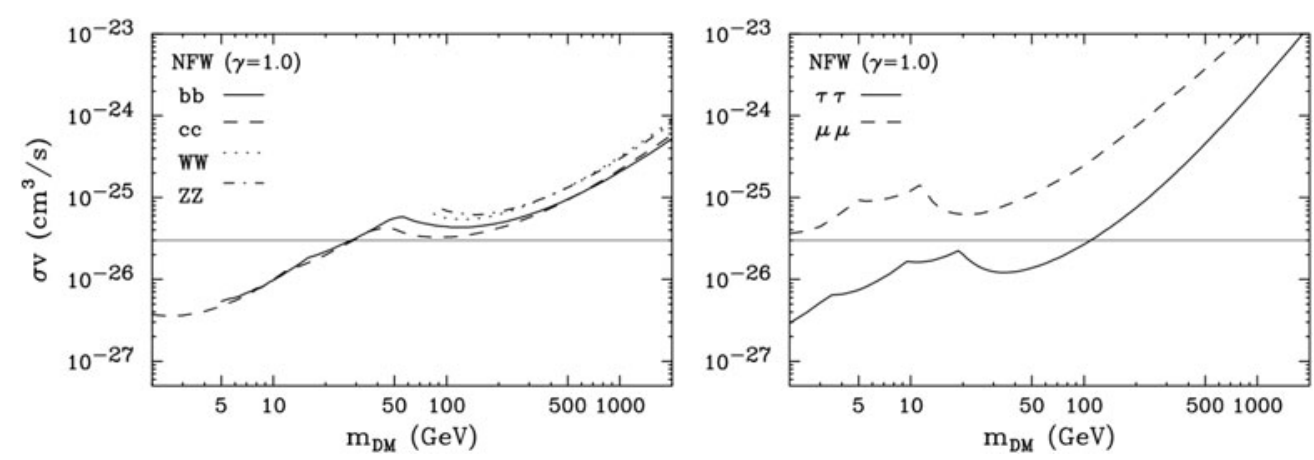

Figure 2. Constraints on the dark matter annihilation cross-section from $\gamma$-ray observations at the Galactic center with the Fermi/LAT telescope. The dark matter profile is calculated employing a slope $\gamma=1$, and is shown for several different annihilation products of dark matter annihilations, including hadronic species on the left, and leptonic species on the right. The horizontal line shows the thermal cross-section which generically produces the correct relic abundance.

only astrophysical assumptions (i.e. Where is the dark matter located? and How far is it from us?). Thus, the expected annihilation flux for dark matter can be compared between different regions of space without regard for the unknown particle physics parameters of our model.

Models almost universally predict that the Galactic center of the Milky Way should provide the brightest signal for dark matter annihilation in the sky, with a predicted luminosity nearly two orders of magnitude above other sources such as the dwarf spheroidal galaxies of the Milky Way, or extragalactic galaxy clusters. This implies that photons from dark matter annihilation should be observed first in the Galactic center region, even if the signal can not be reliably extracted from the complex astrophysical backgrounds. It is an interesting coincidence that the total $\gamma$-ray luminosity of the Galactic center region is approximately equivalent to the expected luminosity from dark matter annihilation for the most naively calculated dark matter models. Specifically, using a value $\alpha=1.0$ for the slope of the dark matter density profile, a value $\sigma \mathrm{v} \sim 3.0 \times 10^{-26} \mathrm{~cm}^{3} \mathrm{~s}^{-1}$ for the annihilation cross-section and a dark matter particle with mass $100 \mathrm{GeV}$ annihilating to pairs of bottom quarks produces approximately $20 \%$ of the total emission observed by the Fermi/LAT within $1^{\circ}$ of the Galactic center. This makes $\gamma$-ray searches, primarily with the Fermi/LAT telescope, among the most exciting observations which could potentially observe, or constrain, a dark matter signal.

By modeling known astrophysical emission mechanisms, and $\gamma$-ray emission correlated with galactic gas, tighter constraints can be set on dark matter models which do not overproduce the total observed $\gamma$-ray emission from the Galactic center. In Figure 2 we show the constraints which observations by the Fermi/LAT can put on dark matter annihilation in the parameter space of the dark matter mass and the annihilation crosssection. We note that the limits on the dark matter annihilation cross-section fall below the predicted thermal cross-section for dark matter masses under $30 \mathrm{GeV}$ annihilating to hadronic final states (left) or under $100 \mathrm{GeV}$ annihilating to pairs of tau-leptons (right). This would naively rule out these types of dark matter models from producing the dark matter in the universe if the dark matter is formed through a thermal process (though many particle physics caveats exist). These limits are currently among the strongest limits on the annihilation rate of dark matter in our universe. 


\section{An excess at the Galactic center?}

Interestingly, measurements first made by Hooper \& Goodenough (2011), and subsequently refined by Hooper \& Linden (2011), Abazajian \& Kaplinghat (2012) and Gordon $\&$ Macias (2013) also found an excess in $\gamma$-ray emission centered around the position of Sgr A*. Notably these observations employed radically different methods for exacting the Galactic center residual: Hooper \& Goodenough (2011) first calculated and subtracted all astrophysical emission, using the spherical and cylindrical symmetry of the problem to extract the spherical excess at the Galactic center. Hooper \& Linden (2011) used gas maps to subtract the dominant $\pi^{0}$ foreground along with the updated position and fluxes of all 2FGL point sources, while Abazajian \& Kaplinghat (2012) and Gordon \& Macias (2013) employed a full template analysis which allowed the amplitudes and spectra of the Fermi diffuse model and foreground point sources to fluctuate in the presence of dark matter and produce a best fit. In each case, the Galactic center residual has emerged as a resilient feature of the model, with a spectrum and intensity which is compatible in each case. The residual signal is characterized by three key features, which will be described in detail in the rest of the section. The Galactic center excess is i) spatially extended compared to the Sgr A* point source, ii) spherically symmetric around the position of the Galactic center, and iii) strongly peaked around an energy of $\sim 2 \mathrm{GeV}$, with a very hard spectrum in the range $300-1000 \mathrm{MeV}$.

The most important astrophysical background of possible dark matter emission from the Galactic center is high energy emission from the supermassive black hole and its surrounding environment. It is worth noting that for $\gamma$-ray observations, the point-spread function of the Fermi/LAT ranges from $0.1^{\circ}$ at the highest energies to $\sim 4^{\circ}$ using a low energy cutoff of $300 \mathrm{MeV}$, which corresponds to $13.9-418 \mathrm{pc}$ at the position of Sgr A*. Thus, the dense region surrounding Sgr $\mathrm{A}^{*}$, including the circumnuclear ring, gaseous outflows, and dense star formation regions, are all included in the $\gamma$-ray point source observed at the Galactic center. Depending on the dark matter density profile, a considerable portion of the $\gamma$-ray signal from dark matter annihilation can also be confined within this region. For the dark matter profile given by Equation 1.1, only $1 \%$ of the total $\gamma$-ray emission from the inner $1 \mathrm{kpc}$ of the Galactic center is confined within the inner $10 \mathrm{pc}$ for the case $\alpha=1.0$, but $41 \%$ of the total $\gamma$-ray emission from the inner $1 \mathrm{kpc}$ is produced within the inner $10 \mathrm{pc}$ for the case $\alpha=1.4$. Therefore it is necessary to use statistical methods to differentiate the dark matter and point source morphologies, since they may differ on angular scales smaller than the instrumental PSF.

This separation has been attempted in two separate ways. Models by Hooper \& Goodenough (2011) produced a source spectrum by fitting the a point source template to the residual emission remaining when the diffuse model and nearby point sources were subtracted, while work by Abazajian \& Kaplinghat (2012) and Gordon \& Macias (2013) and calculated the emission spectrum by employing a best fit template to the total observed emission and allowing all input models, such as the diffuse background, nearby point sources, and the isotropic background, to vary. In both cases the central point source (constrained to the known PSF of the Fermi/LAT instrument) does not provide a good fit to emission very near the Galactic center region. This is perhaps best shown within the context of the fitting algorithms employed by Abazajian \& Kaplinghat (2012), where attempting to produce a model that fits the data using only a point source at the Galactic center significantly over subtracts the emission at the position of the point source, and leaves a ring of bright emission around the Galactic center. This is the expected result if a point source is fit to a source which is actually extended. Using this model, Abazajian \& Kaplinghat (2012) are able to claim an improvement in the log-likelihood of 
nearly 400 with the addition of a template characterized by a spherical spatial extension, a result which (if the error is naively interpreted to be Gaussian) correlates to a $20 \sigma$ preference for an extended residual in the Galactic center. Slight (and ad hoc) alterations to the assumption of spherical symmetry were not found to produce improvements in the log-likelihood compared to the spherically symmetric model. Gordon \& Macias (2013) found that the morphology of the excess is best fit if the three dimensional $\gamma$-ray source is centered at the position of Sgr $\mathrm{A}^{*}$, and falls off in luminosity as $\mathrm{r}^{-2.44 \pm 0.04}$, where $\mathrm{r}$ is the three-dimensional distance from the Galactic center. This corresponds to a dark matter density which falls off as $\mathrm{r}^{-1.22 \pm 0.02}$.

Both modeling efforts which attempt to match the residual to a dark matter signal, (i.e. Hooper \& Goodenough (2011) and Hooper \& Linden (2011)) as well as template fitting algorithms which allow the dark matter component to vary along with relevant astrophysical foregrounds (i.e. Abazajian \& Kaplinghat (2012) and Gordon \& Macias (2013)), find an emission spectrum which is considerably peaked at an energy of $\sim 2-3 \mathrm{GeV}$. In general, template fitting models find a slightly softer spectrum at low-energies, which is reasonable because low-energy photons have poor angular resolution, and are difficult to distinguish between various sources. In models which examine only a dark matter residual, existing point sources are able to soak up much of this emission, while in template fitting models, those photons are better attributed to the dark matter template. These differences are important, since the very hard spectrum below $1 \mathrm{GeV}$ is one of the hardest characteristics of the excess to fit with any astrophysical model. Further inquiries into the low-energy spectrum are currently underway by several groups.

\section{Astrophysical models of the $\gamma$-ray excess}

The peaked spectral feature of the $\gamma$-ray excess is at odds with the majority of astrophysical source templates, which tend to have relatively smooth power-law (or broken power-law) templates stemming from first-order Fermi acceleration. However, the energy of the peak $(2-3 \mathrm{GeV})$ is reminiscent (if not equivalent) to that expected from $\gamma$-rays produced by $\pi^{0}$-decay, where the hard spectrum under $1 \mathrm{GeV}$ is due to the proton energy required to produce a $\pi^{0}$ in the p-p center of mass frame. However, the observed $\gamma$-ray feature is significantly harder than the $\pi^{0}$ decay spectrum which would be produced by any astrophysical (e.g. power-law) distribution of injected protons. In order to produce such a hard spectrum from $\sim 300 \mathrm{MeV}-1 \mathrm{GeV}$, Hooper \& Linden (2011) found that the input proton injection spectrum must be nearly a delta function, at approximately $25 \mathrm{GeV}$, a model that does not correspond to any known class of astrophysical sources.

However, the mechanics of hadronic emission from the central black hole are not well understood, and it is conceivable that the protons may be produced with a characteristic bump at any given energy. Moreover, TeV observations of the Galactic center are fit extremely well by hadronic models, where a population of very high energy protons is emitted from the central point source, and interacts with the dense gas inside the circumnuclear region. This model explains both the lack of variability in $\mathrm{TeV}$ observations, as well as the simple $\mathrm{E}^{-2}$ spectrum observed by H. E. S. S (Aharonian et al. (2006), Chernyakova et al. (2011)).

Models by Chernyakova et al. (2011) note that extending this power-law down to GeV energies could explain the spectrum of the Galactic center point source observed by the Fermi/LAT. Moreover, this model requires a particular set of diffusion parameters near the Galactic center, which features a turnover in the diffusion properties of the regime between $\mathrm{GeV}$ and $\mathrm{TeV}$ energies. Specifically, the propagation of protons was required to be diffusive at $\mathrm{GeV}$ energies, but free-streaming at $\mathrm{TeV}$ energies. This could potentially 
explain the spatial extension in the emission observed at $\mathrm{GeV}$ energies, compared to the point-source nature of the $\mathrm{TeV}$ signal. This is due to the fact that the steady state density distribution for diffusive propagation falls as $\mathrm{r}^{-1}$, compared to $\mathrm{r}^{-2}$ for free-streaming particles.

In order to test this hypothesis, Linden et al. (2012) employed realistic gas models for the Galactic center region produced by Ferrière, K. (2012), and calculated the emission morphology and spectrum produced from protons injected at the Galactic center. This analysis found that the emission morphology is determined primarily by the very peaked gas density in the circumnuclear ring, which lies only 1-3 pc from the position of Sgr A*. Since this region is well within the Fermi/LAT PSF (which is poorer than the H. E. S. S. PSF), any $\gamma$-ray flux produced by protons which are ejected from Sgr A* should appear point-like in the Fermi/LAT analysis. It is thus difficult to imagine a scenario in which a new source of Galactic center protons can produce the $\gamma$-ray excess. Upcoming observations from the Cherenkov Telescope Array should be able to conclusively test this hypothesis, by differentiating $\mathrm{TeV}$ emission from the position of $\mathrm{Sgr} \mathrm{A}^{*}$ from emission at the position of the circumnuclear ring Linden \& Profumo (2012).

Another source class that includes a spectral bump at an energy of $2-3 \mathrm{GeV}$ are $\gamma$-ray pulsars. The second Fermi/LAT catalog of $\gamma$-ray pulsars detects 117 such sources Abdo et al. (2013) including populations of milli-second pulsars (MSPs), and blind $\gamma$-ray pulsars (i.e. pulsars first not detected in any other wavelength). None of these sources exist very close to the position of the Galactic center. However, this is not unexpected because the blind detection of pulsars is difficult, especially in regions where the source may be accelerating (such as within binaries or near the Galactic center). While young pulsars can provide a high $\gamma$-ray luminosity, it is difficult to employ young pulsars to explain the Galactic center excess, since their morphology should follow (or be more diffuse than) the morphology of massive stars near the Galactic center. The observed stellar distribution is well constrained observation and is significantly more diffuse than the $\mathrm{r}^{-2.4}$ distribution of the $\gamma$-ray excess. While MSPs are comparatively dim, Abazajian 2011 pointed out that a population of $\sim 1000$ MSPs could produce the $\gamma$-ray luminosity observed at the Galactic center. While the distribution of young pulsars is expected to follow the stellar density, MSPs may follow the square of the stellar density if they are formed (and spun up) after binary interactions with other stellar systems. This phenomenon is observed in the related low-mass X-Ray Binary population of M31 Voss \& Gilfanov (2007). Work by Abazajian \& Kaplinghat (2012) and Gordon \& Macias (2013) found that MSPs produce a good fit to the spectrum of the observed excess, with the best fitting log-parabola spectrum approximately $1 \sigma$ offset from the average of MSPs observed by the Fermi/LAT. It is worth noting that very recent measurements of a Magnetar near the Galactic center by the NuSTAR experiment, and the subsequent constraints on the dispersion measurement by follow-up radio observations, may put strong constraints on the possible population of MSPs very close to the Galactic center Mori et al. (2013). Current models would predict the observation of pulsations from at least two MSPs, while none have yet been observed Spitler et al. (2014).

Recently, observations by Hooper \& Slatyer (2013) pointed towards the existence of a similar spectral bump at approximately $2-3 \mathrm{GeV}$ in the inner Galaxy region (a region we will define as approximately 10-20 degrees away from the Galactic center and spherically distributed around the plane). While a thorough comparison of this signal with that detected closer to the Galactic center is currently underway, Hooper et al. (2013) points out that if these are in fact the same signal, the high luminosity observed in the inner Galaxy would exclude MSPs as a source of the emission. In order to normalize the luminosity distribution of MSPs in order to fit the total emission in the inner Galaxy, 

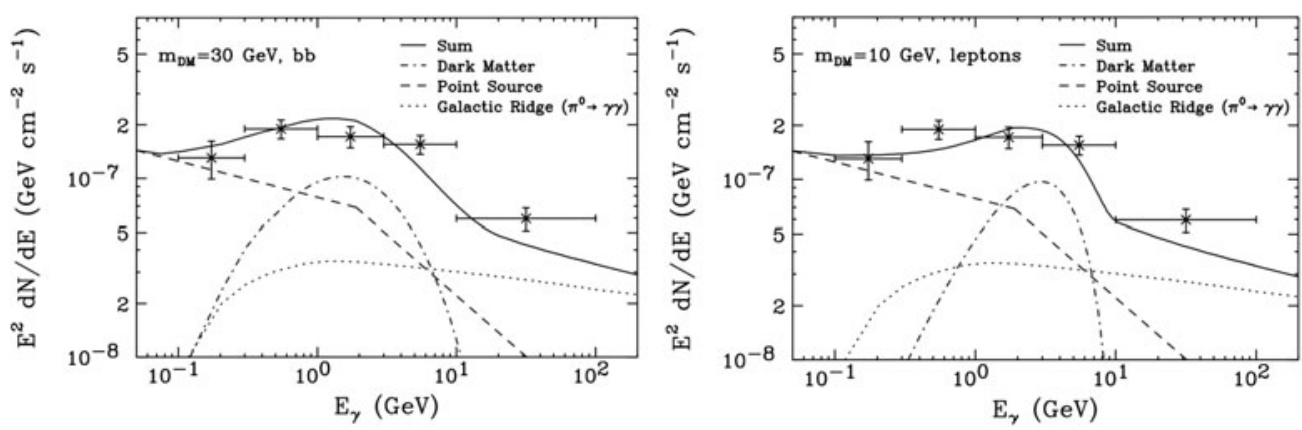

Figure 3. Spectral Fits from Dark Matter Annihilation to the $\gamma$-ray signal observed within $5^{\circ}$ of the Galactic center, once emission from known point source (except for the Galactic center point source) and emission correlating with galactic gas is removed. Dark matter models corresponding to a $30 \mathrm{GeV}$ particle annihilating to $b \bar{b}$ (left) and a $10 \mathrm{GeV}$ particle annihilating democratically to all lepton generations (with $\tau^{+} \tau^{-}$dominating the signal; right) both produce a reasonable fit to the excess. Recent studies by Abazajian \& Kaplinghat (2012) and Gordon \& Macias (2013) find the fit from $\sim 30 \mathrm{GeV}$ particles annihilating to $b \bar{b}$ to be statistically preferred to the leptonic cases.

too many bright (and detectable) MSPs would have to be produced. Even if pulsations from these sources were not detected, the Fermi/LAT would observe these MSPs as unassociated $\gamma$-ray sources. Constraining the number of bright MSPs to the total number of unassociated sources in the emission region, and then using a luminosity distribution for MSPs in order to calculate the total emission from dimmer sources, under-produces the excess observed by Hooper \& Slatyer (2013) by more than an order of magnitude. This is currently the strongest evidence against MSPs producing the Galactic center signal.

\section{Dark matter models of the $\gamma$-ray excess}

Several dark matter models have also been produced in order to account for the emission observed in the Galactic center. The parameters of these models are generically set by the need to produce a spectral bump at the required energy of $2-3 \mathrm{GeV}$. This roughly corresponds to the spectrum expected from a $30 \mathrm{GeV}$ dark matter particle annihilating to hadronic final states, (with a matter-antimatter pair of bottom quarks $(b \bar{b})$ usually taken as a generic model), or alternatively, a lighter dark matter particle with mass $8-10 \mathrm{GeV}$ annihilating primarily to $\tau+\tau-$ leptons, which produce a harder $\gamma$-ray spectrum. In Figure 3 we show the calculated spectrum from these models, including a broken power-law astrophysical background which corresponds to the best fitting spectrum for the Sgr A* point source, and a second power-law background corresponding to an extrapolation of the H. E. S. S. ridge observed at TeV energies Aharonian et al. (2006). In both cases, we find a reasonable match to the spectrum of the $\gamma$-ray residual.

In some sense, these dark matter models are relatively easy to fit to any bump in the data. For instance, if the $\gamma$-ray spectrum were to produce a bump at 6-9 $\mathrm{GeV}$ (with similar spectral slopes as the observed bump), dark matter models with masses three times the above region would likely produce reasonable fits. On small scales close to the Galactic center, the morphology of the excess is also relatively unconstrained, though a reasonable benchmark is a dark matter density falling $\mathrm{r}^{-1}$, which corresponds to an annihilation rate falling as $\mathrm{r}^{-2}$. However, theoretical models have been written down here the annihilation rate varies from being constant with the Galactic center distance to falling as $\mathrm{r}^{-3}$ (Governato et al. (2011), Gnedin et al. (2011)). Somewhat more convincing 


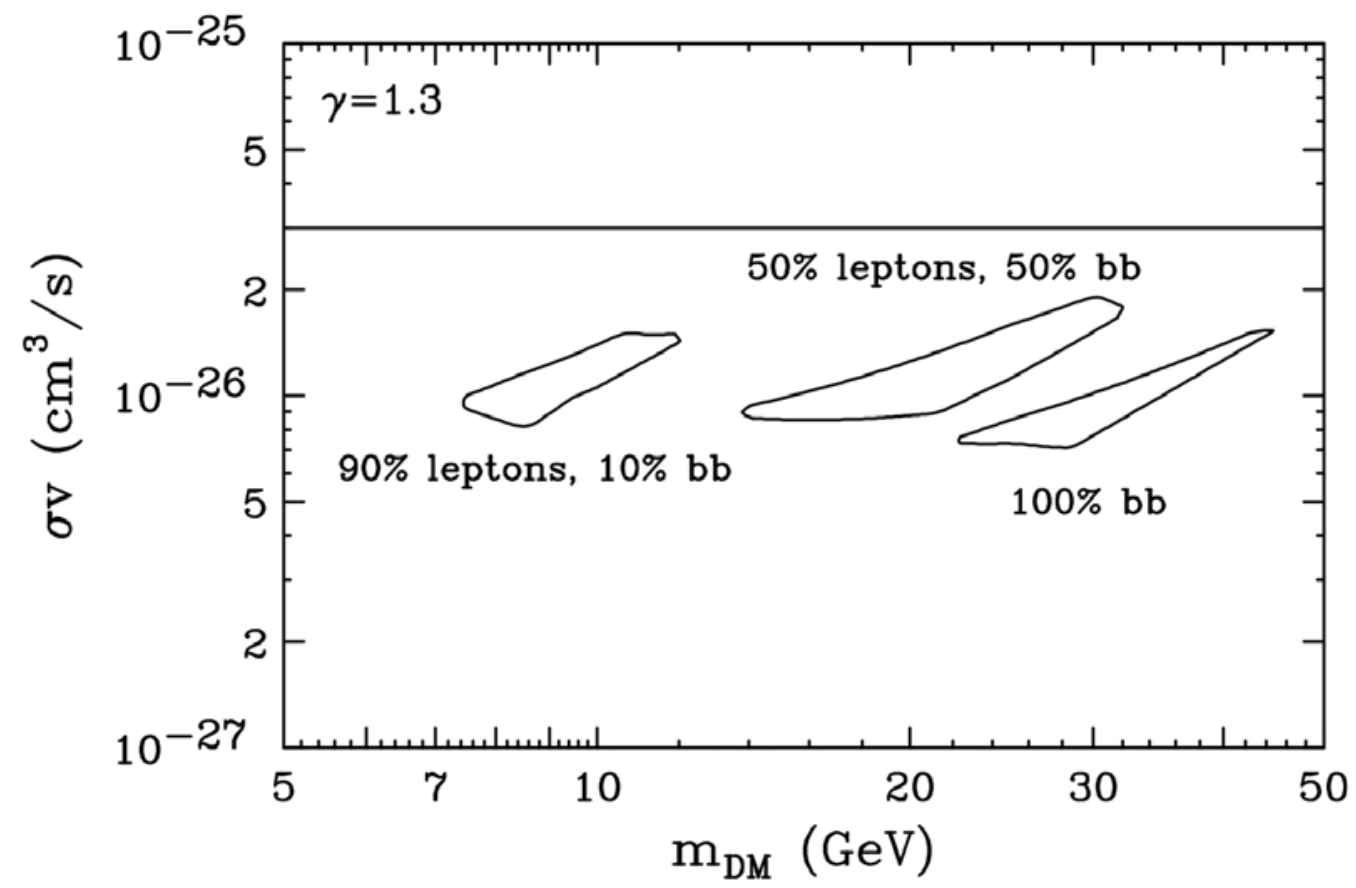

Figure 4. Dark Matter cross-sections $(\sigma \mathrm{v})$ and match regions, which provide the optimal fit to the $\gamma$-ray excess in the Galactic center. The values of the fit are shown for a dark matter density profile which goes as $\mathrm{r}^{-1.3}$. The primary effect of using a $\mathrm{r}^{-1.2}$ profile would be an increase in the calculated cross-section by approximately a factor of three.

is the spherical symmetry of the residual, which is a generic prediction of collisionless dark matter profiles, although astrophysical source classes may also show spherical symmetry very near the Galactic center.

Finally, it is worth noting that the necessary cross-section for dark matter is within the range expected for the WIMP miracle. In Figure 4 we show the cross-section and mass range which provide the best fits to the observed excess. The horizontal line across the plot shows the expected thermal relic cross-section. While our models fall slightly below this value, this can easily be accommodated when particle physics uncertainties are included in the model. For instance, the dark matter particle may also annihilate to other final states, such as neutrinos, which do not provide any $\gamma$-ray signal but do contribute to the thermal cross-section. Furthermore, our fits utilized a dark matter density profile which falls as $\mathrm{r}^{-1.3}$, while more recent studies such as Gordon \& Macias (2013) prefer a profile of $\mathrm{r}^{-1.22}$, which boosts the cross-sections by approximately a factor of three.

\section{Multi-wavelength signals from dark matter at the Galactic center}

In addition to $\gamma$-ray signals, dark matter annihilation should inject an energetic population of high energy hadrons and leptons into the interstellar medium. The energy losses of these particles can produce significant bremsstrahlung, inverse-Compton scattering, and synchrotron emission. Inverse-Compton scattering and bremsstrahlung emission from $\mathrm{GeV}$-scale particles are likely to also produce $\gamma$-ray photons that contribute to the total $\gamma$-ray signal from dark matter (see e.g. Cirelli et al. (2013)). However, synchrotron emission is likely to produce a significant radio signal in the Galactic center. 
This is especially true in the case where dark matter annihilates primarily to leptonic pairs.

Interestingly, several significant radio features exist in the Galactic center region, which are currently difficult to explain with astrophysical mechanisms. Most notably, emission from the filamentary arcs near the Galactic center have an extremely hard synchrotron spectrum which is best fit if the injected electron spectrum is approximately a delta function of approximately $10 \mathrm{GeV}$ Lesch et al. (1998). Dark matter annihilating equally to all lepton generations produces such a spectrum, and Linden et al. (2011) showed that dark matter annihilating with the cross-section and morphology indicated by the above $\gamma$-ray excess could naturally explain the spectrum and intensity of multiple filamentary arcs. Efforts are currently underway to understand whether additional excesses in lowenergy Galactic center emission, such as the $\mathrm{K} \alpha$ line, may be explained by electrons produced via dark matter annihilation.

Finally, it is worth noting that several direct detection experiments also currently point to a light dark matter particle with a mass of 8-10 GeV (Aalseth et al. (2011), Angloher et al. (2012) ). However, these observations are also significantly ruled out by other direct detection experiments Ahmed et al. (2011), Aprile et al. (2011), Akerib et al. (2013) . This is an experimental question which should be resolved within the coming years, and may make the current signal in the Galactic center significantly more exciting to dark matter hunters.

\section{Conclusions}

The Galactic center continues to be a particularly exciting target for searches of dark matter annihilation, since the high flux expected from this region implies that the indirect detection of dark matter from any astrophysical target should be at least compatible with the observed $\gamma$-ray flux at the Galactic center. Future observations which more carefully consider both the astrophysical $\gamma$-ray backgrounds in the Galactic center and also examine the multi-wavelength constraints on both astrophysical and dark matter sources will greatly clarify the status of these observations. The instrumental capabilities of telescopes such as the Fermi/LAT have taken us to a point where we can observe or constrain the most generic dark matter models which were written down long before dark matter annihilation could be conceivably probed. The next decade promises to be an exciting time in the search for dark matter annihilation, and the Galactic center stands at the forefront for indirect dark matter searches.

\section{References}

Aalseth, C., et al. 2011, Phys. Rev. Lett. 106, 131310

Abazajian, K., 2011, JCAP 3, 10

Abazajian, K. \& Kaplinghat, M. 2012, Phys. Rev. D 86, 8

Abdo, A. A., et al. 2013, ApJS 208, 17

Aharonian, F., et al. 2006, ApJ 636, 777

Aharonian, F., et al. 2006, Nature 439, 695

Ahmed, Z., et al. 2011, Phys. Rev. Lett. 106, 131302

Akerib, D., et al. 2013, arXiv: 1310.8214

Angloher, G., et al. 2012, European Physics Journal 72, 1971

Aprile, E., et al. 2011, Phys. Rev. Lett. 107, 131302

Chernyakova, M., Malyshev, D., Aharonian, F. A., Crocker, R., \& Jones, D. 2011, ApJ 726, 60

Cirelli, M., Serpico, P., \& Zaharijas, G. 2013, JCAP 11, 35

Diemand, J., et al. 2008, Nature 454, 735 
Ferrière, K. 2012, A\&A 540, 50

Gnedin, O. Y., Ceverino, D., Gnedin, N. Y., Klypin, A. A., Kravtsov, A. V., Levine, R., Nagai, D., \& Yepes, G. 2011, arXiv 1108.5736

Gordon, C. \& Macias, O. 2013, Phys. Rev. D 88, 8

Governato, F., Zolotov, A., Pontzen, A., Christensen, C., Oh, S. H, Brooks, A. M., Quinn, T., Shen, S., \& Wadsley, J. 2012, MNRAS 422, 1231

Hooper, D., Cholis, I., Linden, T., Siegal-Gaskins, J., \& Slatyer, T. 2013, Phys. Rev. D 88, 8

Hooper D. \& Goodenough, L. 2011, Phys. Lett. B 697, 412

Hooper, D. \& Linden, T. 2011, Phys. Rev. D 84, 12

Hooper, D. \& Slatyer, T. 2013, Physics of the Dark Universe 2, 118

Iocco, F., Pato, M., Bertone, G., \& Jetzer, P. 2011, JCAP 029, 29

Lesch, H, Schlickeiser, R. \& Crusius, A. 1988, A\&A 200, L9

Linden, T., Hooper, D., \& Yusef-Zadeh, F. 2011, ApJ 741, 95

Linden, T., Lovegrove, L., \& Profumo, S. 2012, ApJ 753, 41

Linden, T. \& Profumo, S. 2012, ApJ 760, 23

McMillan, P. J. 2011, MNRAS 414, 2446

Mori, K. 2013, ApJ Lett. 770, L23

Navarro, J., Frenk, C., \& White, S. 1996, ApJ 462563

Navarro, J., et al. 2004, MNRAS 349, 1039

Pagels, H. \& Primack, J. 1982, Phys. Rev. Lett. 48223

Spitler, L., et al. 2014, ApJ Lett. 780 L3

Steigman, G. 1979, Ann. Rev. of Nuclear and Particle Science 29313

Voss, R. \& Gilfanov, M. 2007, A\&A $46849 \mathrm{~V}$ 